\title{
Progressive Medullary Congestion Resulting in Life-Threatening Condition Caused by Lower Cervical Dural Arteriovenous Fistula Successfully Treated with Endovascular Treatment: A Case Report and Literature Review
}

\section{Prasert lampreechakul (D) ${ }^{*}$, Punjama Lertbutsayanukul ${ }^{2}$, Yodkhwan Wattanasen ${ }^{2}$ and Somkiet Siri- wimonmas ${ }^{3}$}

${ }^{1}$ Department of Neurosurgery, Prasat Neurological Institute, Bangkok, Thailand

${ }^{2}$ Department of Neuroradiology, Prasat Neurological Institute, Bangkok, Thailand

${ }^{3}$ Department of Radiology, Bumrungrad International Hospital, Bangkok, Thailand

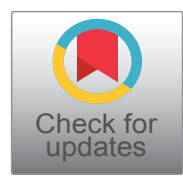

*Corresponding author: lampreechakul P, Department of Neurological Surgery, Prasat Neurological Institute, 312 Ratchawithi Road, Khwaeng Thung Phaya Thai, Bangkok 10400, Thailand, Email: Bangruad@hotmail.com

\begin{abstract}
The authors describe a patient with lower cervical dural arteriovenous fistula (DAVF), subsequently developing brainstem dysfunction following progressive myelopathy caused by extension of venous congestion from the cervicothoracic cord to the medulla oblongata. A 34-year-old woman suffered from progressive paraparesis with bowel/bladder dysfunction for 2 months. Before transferring to our institute, she obtained magnetic resonance imaging (MRI) of the neck, showing abnormal hyperintensity on T2-weighted sequence extending from the level of $\mathrm{C} 1$ to $\mathrm{T} 5$ with abnormal intradural flow voids along anterior more than posterior pial surfaces of the cervical spinal cord. Due to clinical deterioration, the patient was admitted before the schedule and obtained follow-up MRI, revealing progression of abnormal hyperintensity extending from the level of T7 to the lower medulla, representing progressive venous congestion. Contrast-enhanced magnetic resonance angiography (MRA) demonstrated a cervical DAVF at the C8 nerve root, supplied by a feeder originating from the deep cervical artery, and prominently engorged draining veins extending rostrally to the pial venous network around the medulla, and caudally to the thoracic cord. There was a venous pouch located in the right C7-T1 intervertebral foramen. During waiting for endovascular treatment, the patient developed respiratory dysfunction, needed endotracheal intubation. Emergency embolization was successfully performed with $\mathrm{N}$-butyl-2-cyanoacrylate. $\mathrm{MRI}$ and angiography of the cervical spine, obtained few
\end{abstract}

months after embolization, confirmed complete obliteration of the fistula and resolution of venous congestion. Three years follow-up MRI and MRA showed no recurrence of the fistula. The patient still had spastic paraparesis. Bowel and bladder dysfunction had gradually improved. Natural history of spinal DAVFs in lower cervical spine probably has more aggressive clinical course than thoracolumbar region. Prompt treatment should be performed before clinical deterioration due to brainstem dysfunction. Delayed treatment resulted in irreversible neurological deficit.

\section{Keywords}

Lower cervical spine, Spinal dural arteriovenous fistula, Brainstem congestion, Venous congestion, Medulla oblongata, Brainstem dysfunction

\section{Introduction}

Spinal dural arteriovenous fistulas (SDAVFs) are the most common type of spinal vascular malformations. They usually become symptomatic in middle-aged men aged between 40-60 years and occur in the thoracolumbar region. SDAVFs, also referred to as type I spinal AVMs, and intradural dorsal AVFs, are the shunts supplied by radiculomeningeal arteries draining into the radicular veins arterializing the coronal venous plexus around the spinal cord (perimedullary veins), usually

Citation: lampreechakul P, Lertbutsayanukul P, Wattanasen Y, Siriwimonmas S (2019) Progressive Medullary Congestion Resulting in Life-Threatening Condition Caused by Lower Cervical Dural Arteriovenous Fistula Successfully Treated with Endovascular Treatment: A Case Report and Literature Review. Clin Med Rev Case Rep 6:275. doi.org/10.23937/2378-3656/1410275

Accepted: July 23, 2019: Published: July 25, 2019

Copyright: (c) 2019 lampreechakul P, et al. This is an open-access article distributed under the terms of the Creative Commons Attribution License, which permits unrestricted use, distribution, and reproduction in any medium, provided the original author and source are credited. 
under low pressure, resulting in venous congestion. The fistulas usually locate intradural dorsal aspect close to the root sleeves. The patients typically manifest with gradual progression of myelopathy induced by chronic venous hypertension [1-3]. Intramedullary hemorrhage secondary to SDAVFs is extremely rare, and usually causes by venous varix [4]. The patients with SDAVFs may present with subarachnoid hemorrhage $(\mathrm{SAH})$, especially in the region of the craniocervical junction [57]. Brainstem congestion due to SDAVFs is also tremendously rare, and was reported at the craniocervical region $[8,9]$. Cervical DAVFs can be subdivided into upper, i.e., craniocervical junction or $\mathrm{C} 1-\mathrm{C} 2$, and lower levels, i.e., below $\mathrm{C} 2$ [10]. The authors described a young woman with lower cervical DAVF manifested with brainstem dysfunction following progressive myelopathy caused by extension of venous congestion from cervicothoracic cord to medulla oblongata. Endovascular treatment was performed in the emergency setting, and the fistula was successfully obliterated using small amount of $\mathrm{N}$-butyl-2-cyanoacrylate (NBCA). In addition, the authors also reviewed the literature of lower cervical DAVF (C3-C8).

\section{Case Report}

A 34-year-old woman primarily suffered from neck pain radiating to both shoulder blades. There was no underlying disease and history of trauma. One week later, she noticed unsteady gait with urinary retention. She went to local hospital and was admitted for observation and investigation. During hospital course, she had weakness and paresthesia of both legs with constipation. Lumbar puncture was performed, and cerebrospinal fluid analysis showed no abnormality. The doctor probably gave her steroid injections. She markedly improved and discharged home one week later. Two week later, the patient developed quadriparesis with bladder and bowel symptoms. She went back to the local hospital and obtained magnetic resonance imaging (MRI) of the neck, showing abnormal hyperintensity on T2-weighted sequence extending from the level of $\mathrm{C} 1$ to T5 with abnormal intradural flow voids along anterior more than posterior pial surfaces of the cervical spinal cord (Figure 1A). The diagnosis was cervical DAVF. One month later, the patient was transferred to Prasat Neurological Institute (PNI) for endovascular treatment. The neurological examination revealed evidence of spastic quadriparesis (Medical Research Council (MRC) scale for muscle strength grade 4), the lack of pinprick sensation below L1 level, hyperreflexia of upper and lower extremities, clonus and presence of Babinski's sign. Endovascular treatment was scheduled in next month due to a large volume of an interventional neuroradiology

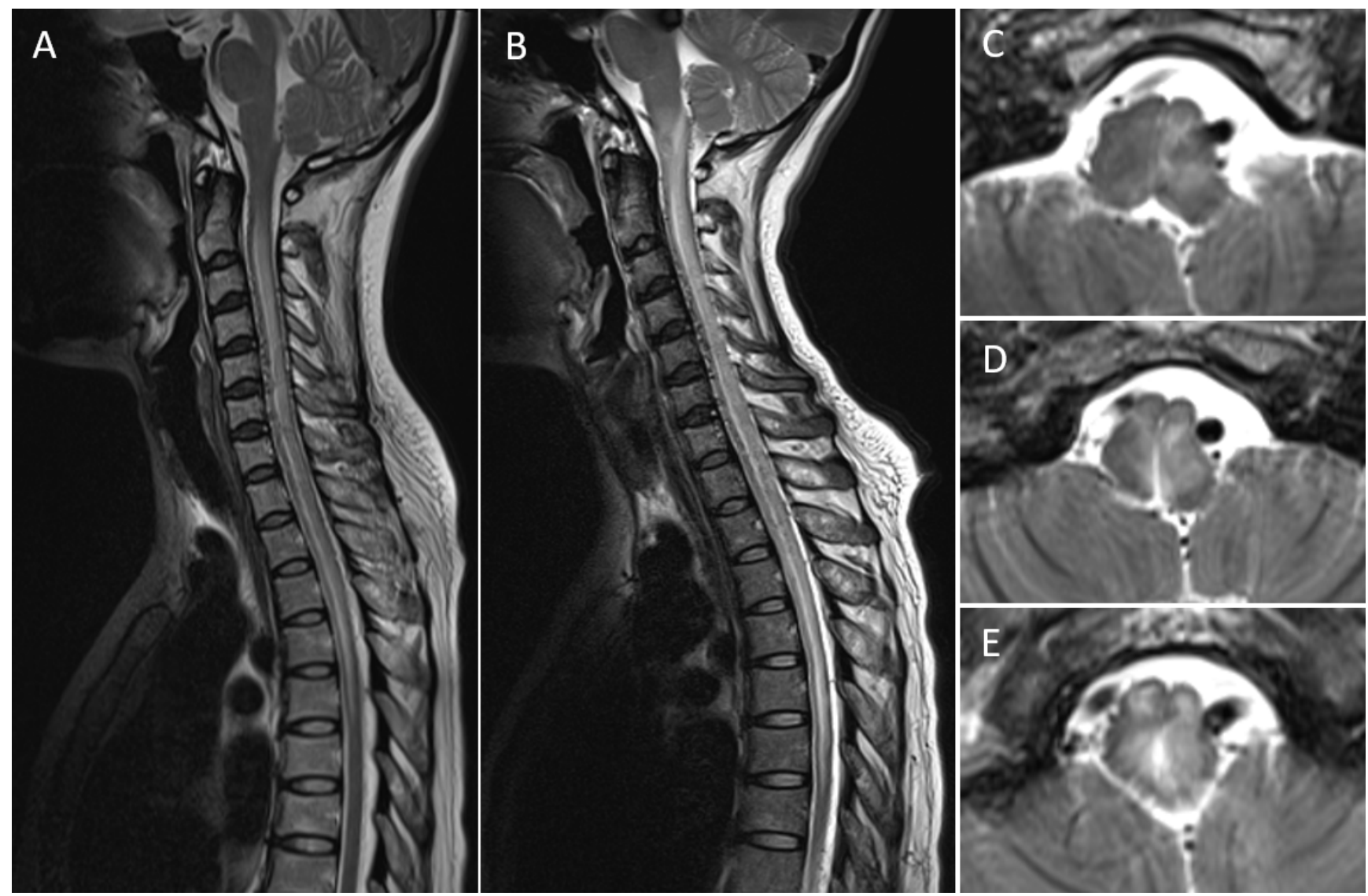

Figure 1: Sagittal T2-weighted MR images obtained 1 months $(A)$ and 3 months $(B)$ after initial symptoms show progression of abnormal hypersignal intensity from cervicothoracic spinal cord to the lower medulla, representing progressive venous congestion. There are abnormal flow voids along ventral more than dorsal surfaces of the cervical spinal cord. Axial T2weighted MR images $(C, D, E)$ from upper to lower level of the medulla demonstrate symmetrically diffuse medullary swelling with subtle flow voids around the dorsal surface of the medulla. 
service in our tertiary referral center. Three weeks later, she experienced severe neck pain radiating to right shoulder blade and suboccipital area, chest pain, and quadriparesis (MRC scale grade 3). She was admitted to

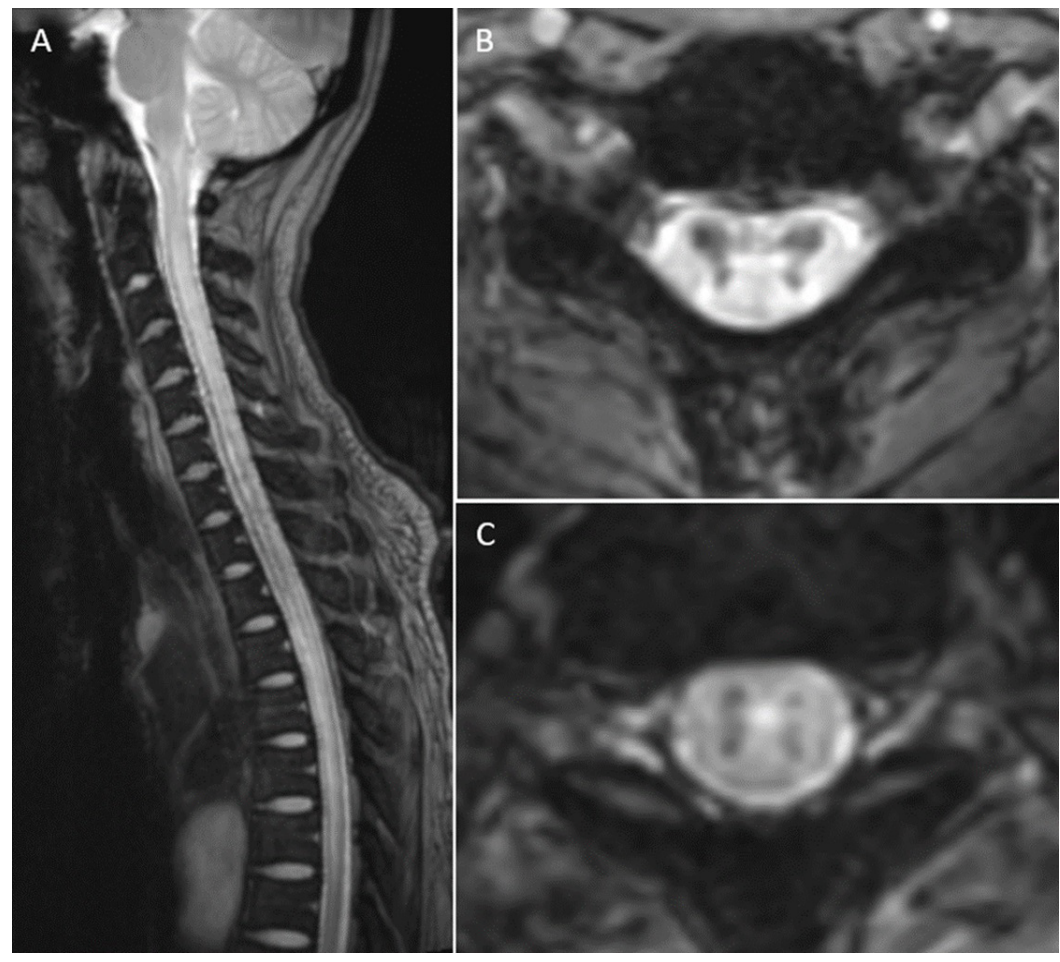

Figure 2: Sagittal (A) and axial gradient echo T2*-weighted images at the level of C5 (B) and T2 (C) of the cervical spine show hypersignal intensity from the medulla to the mid thoracic cord, representing venous congestion. There also is abnormal hyposignal intensity affected predominantly in the central gray matter of the cervical cord from C4 to T3, presumed to be hemosiderin stain from hemorrhage. These findings on axial views resemble the black butterfly silhouette.

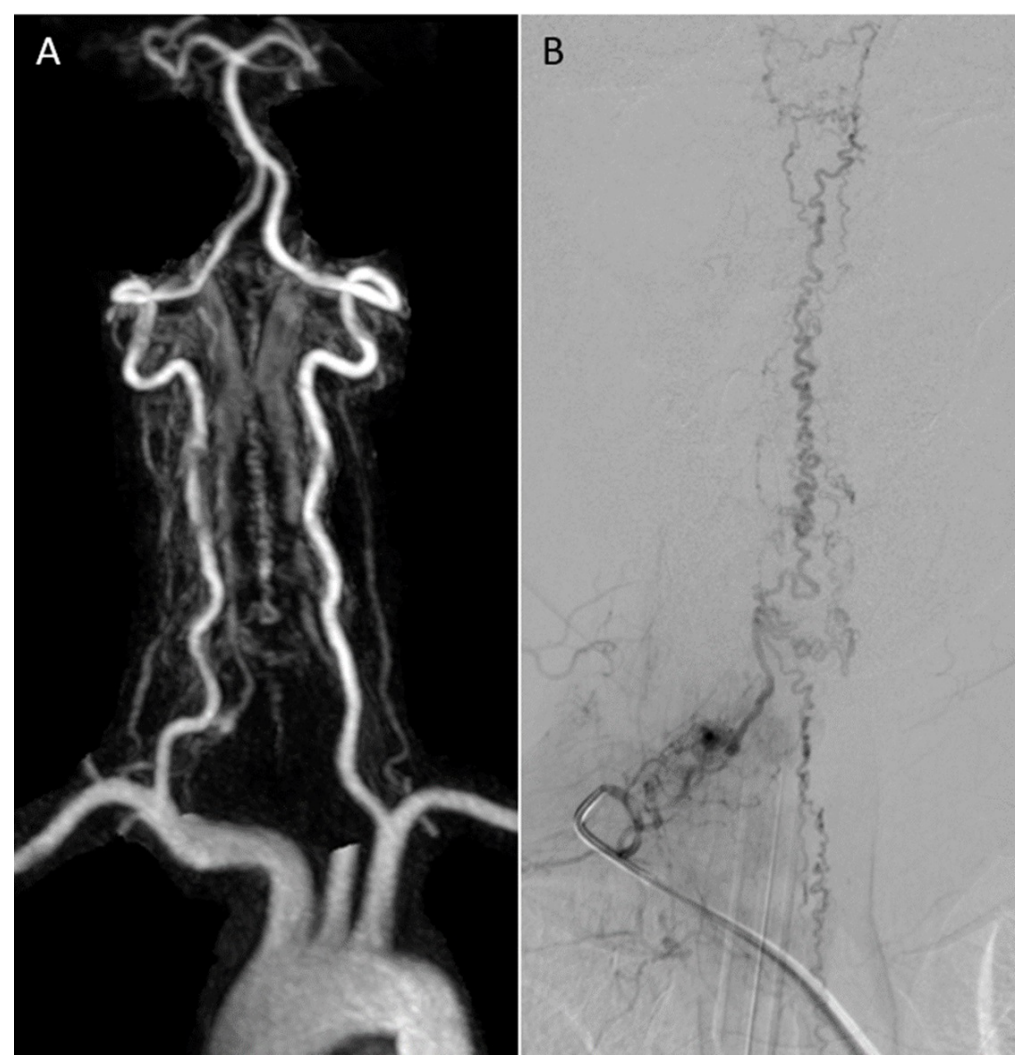

Figure 3: Anteroposterior views of contrast-enhanced magnetic resonance angiography of the neck (A) and spinal angiography of the right costocervical artery injection (B) show a cervical dural arteriovenous fistula with a large feeder originating from the deep cervical artery, and prominently engorged draining veins extending rostrally to the pial venous network around the medulla, and caudally to the thoracic cord. 
PNI for observation and obtained MRI of the neck three days later. T2-weighted image revealed progression of abnormal hyperintensity extending from the level of T7 to lower medulla, representing progressive venous con-
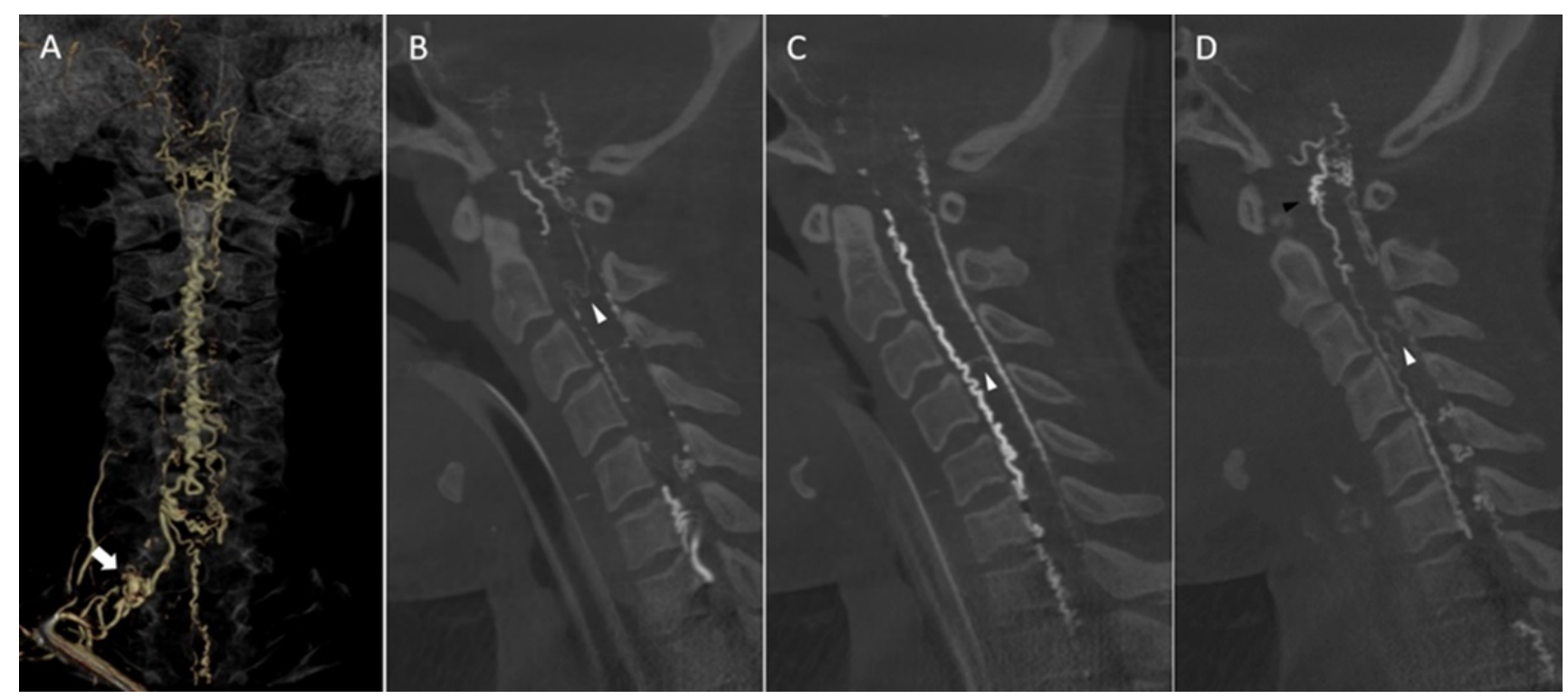

Figure 4: Anteroposterior view of three-dimensional reconstructed image of osseous and vascular structures obtained from rotational angiography of a right costocervical trunk reveals spinal dural AVF with a venous pouch (white arrow) in a right C7-T1 intervertebral foramen, which drains into a right radicular vein and dilated, tortuous coronal venous plexus. Sequential images from right to left side of sagittal maximum intensity projection reformatted images of angiographic computerized tomography of the cervical spine demonstrate dilated, tortuous anterior and posterior median spinal veins. There is longitudinally oriented extrinsic venous network, i.e., perimedullary anastomosis, connecting ascendingly between the dilated lateral anterior spinal vein and dorsal medullary venous plexus (black arrowhead) noted. Also, transmedullary venous anastomoses bridging between anterior and posterior spinal veins (white arrowheads) are clearly demonstrated.

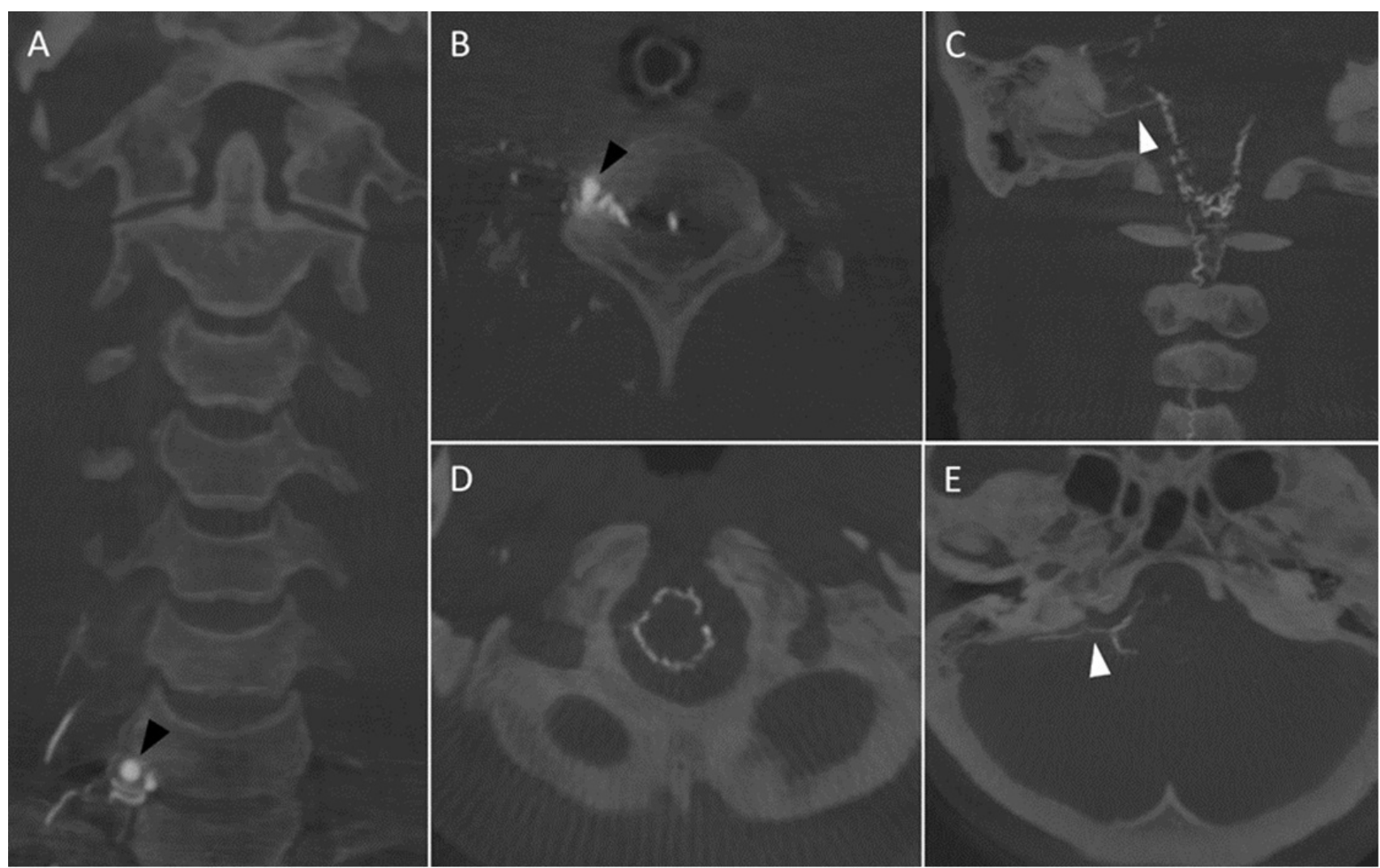

Figure 5: Coronal (A) and axial (B) maximum intensity projection (MIP) reformatted images of angiographic computerized tomography (CT) of the cervical spine reveal fistulous site and a venous pouch in right C7-T1 intervertebral foramen with a dilated draining radicular vein. Coronal $(C)$ and axial (D, E) MIP reformatted images of angiographic CT of the craniocervical junction demonstrate the dilated pial venous network surrounding the surface of the medulla oblongata. There is a bridging vein communicating from the vein of pontomedullary sulcus to dural venous sinus noted. 
gestion (Figure 1B, Figure 1C, Figure 1D, and Figure 1E). Additionally, gradient echo $\mathrm{T} 2^{*}$-weighted image (GRE $\mathrm{T} 2^{*} \mathrm{WI}$ ) disclosed abnormal hyposignal intensity affected predominantly in the central gray matter of the cervical cord from C4 to T3, presumed to be hemosiderin stain from hemorrhage (Figure 2). Contrast-enhanced magnetic resonance angiography (MRA) demonstrated a cervical DAVF supplied by a feeder originating from the deep cervical artery, and prominently engorged draining veins extending rostrally to the pial venous network around the medulla, and caudally to the thoracic cord (Figure 3A). Endovascular treatment was scheduled on the next day. Unfortunately, she developed chest pain, quadriparesis (MRC scale grade 1) and shortness of res- piration on the next morning. The patient was intubated due to developing of hypoxemia. During intubation, there was no gag reflex. Endovascular treatment was performed in the emergency setting. Spinal angiography of the right costocervical artery injection confirmed the fistula as seen in MRA (Figure 3B). In addition, angiographic computerized tomography in three-dimensional reconstructed image and maximum intensity projection (MIP) reformatted images of the cervical spine was obtained and clearly demonstrated a venous pouch in a right C7-T1 intervertebral foramen and venous anastomotic system communicating the cervical spinal cord to the medulla oblongata. Also, MIP reformatted images delineated the dilated pial venous network surrounding

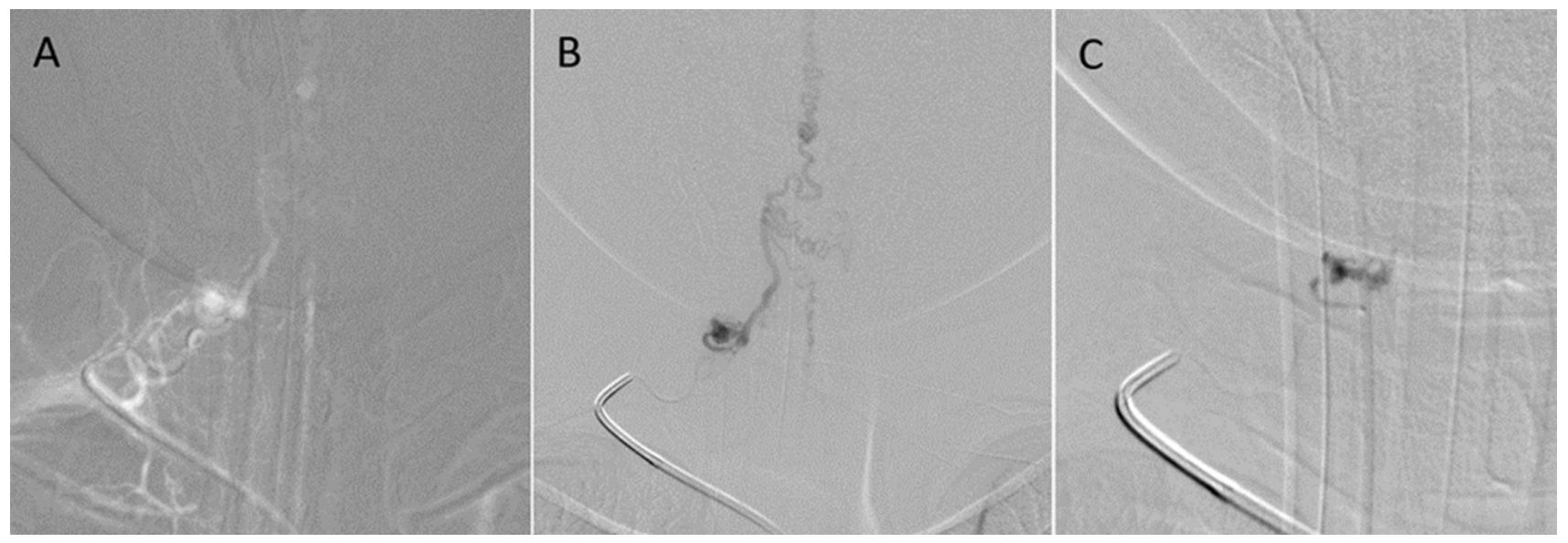

Figure 6: Anteroposterior (AP) view of the right costocervical artery injection using real-time digital subtraction fluoroscopic mapping (A) shows a microcatheter placed in the right deep cervical artery. Selective angiographic AP image of a right deep cervical artery $(B)$ demonstrates a dural arteriovenous fistula with drainage through the radicular vein rostrally and caudally into the coronal venous plexus. There is a dilated venous pouch noted. (C) During embolization with N-butyl-2cyanoacrylate, the venous pouch and proximal draining vein are occluded.

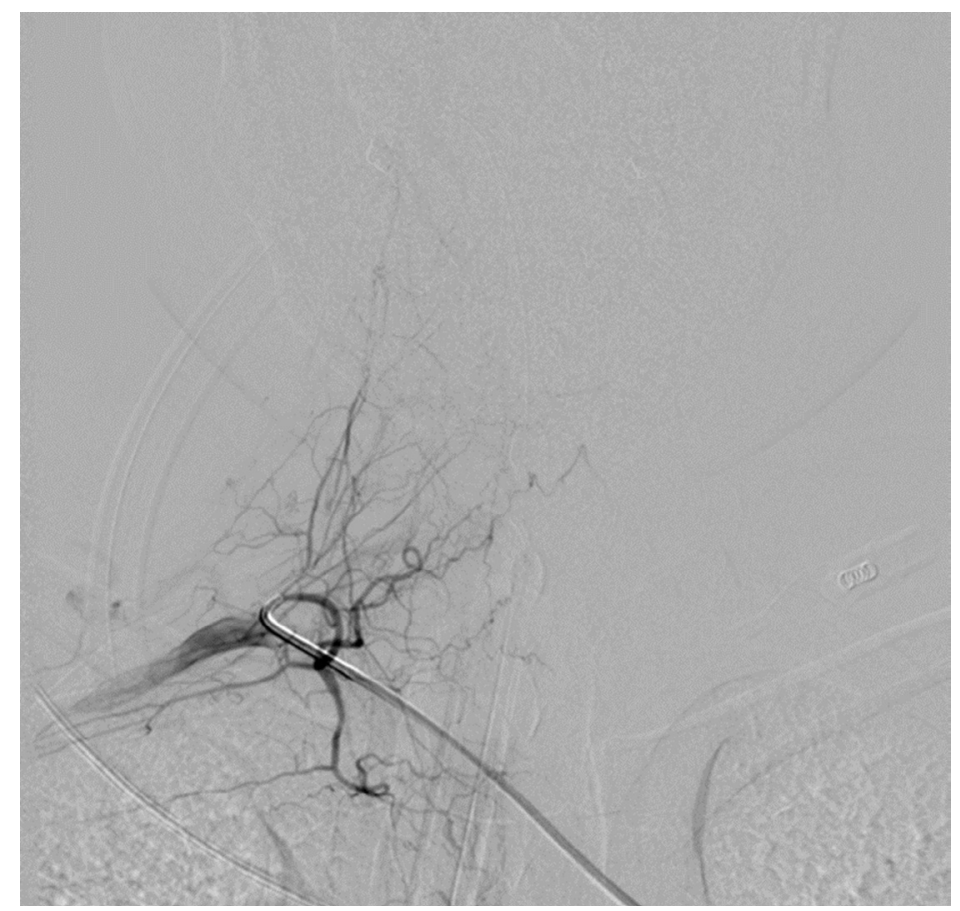

Figure 7: Selective digital subtraction angiography of a right costocervical trunk, obtained 2 months after embolization, confirms complete obliteration of the dural arteriovenous fistula. 


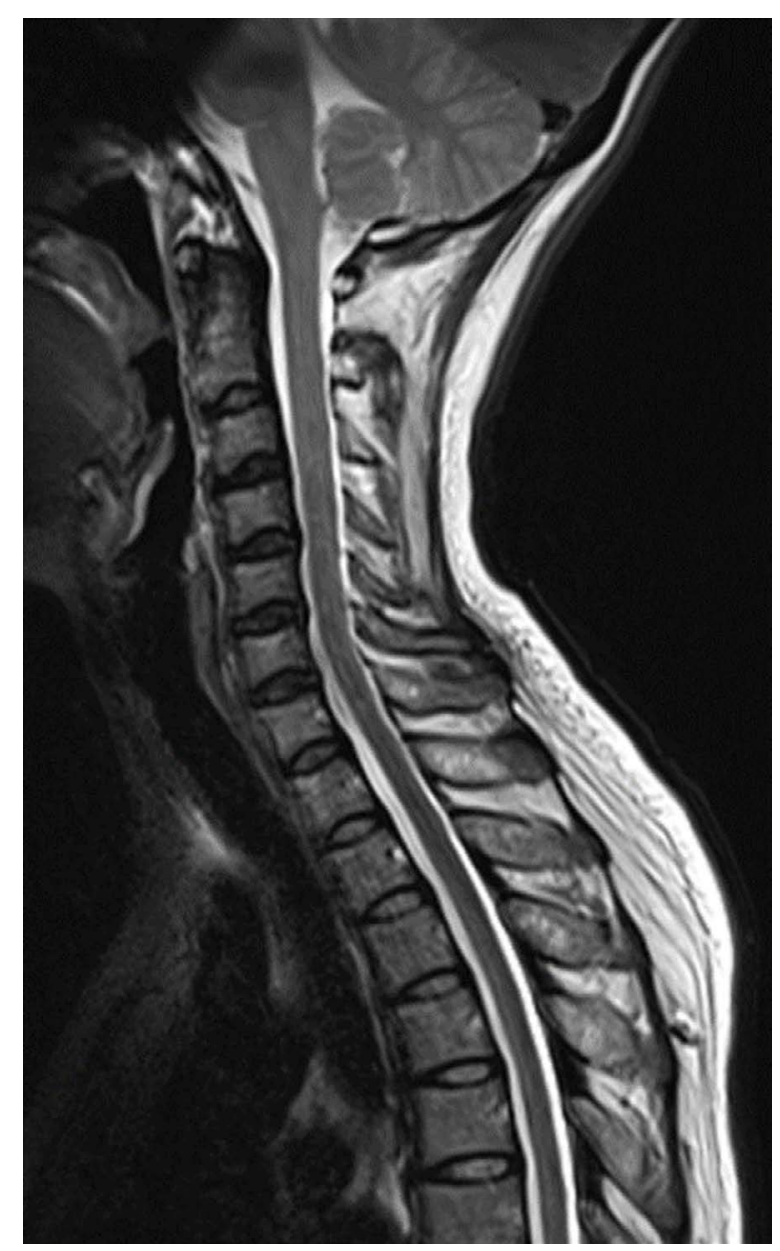

Figure 8: Sagittal T2-weighted magnetic resonance imaging of the cervical spine, obtained 4 months after embolization, confirms complete resolution of venous congestion from the medulla to mid thoracic cord and disappearance of intradural flow voids. Mild spinal cord atrophy is observed.

the surface of the medulla oblongata, and a bridging vein connecting the vein of pontomedullary sulcus to the sigmoid sinus (Figure 4 and Figure 5). Using transarterial embolization through the right deep cervical artery under real-time digital subtraction fluoroscopic mapping, complete occlusion of the proximal draining vein and venous pouch was achieved with small amount of N-butyl-2-cyanoacrylate (NBCA) (Figure 6). The patient was extubated the following day. The power of her upper extremities had rapidly regained. Follow-up angiography, obtained 2 months after embolization, confirmed complete obliteration of the fistula (Figure 7). MRI of the cervical spine, obtained 4 months after embolization, showed complete resolution of venous congestion from the medulla to mid thoracic cord and disappearance of intradural flow voids (Figure 8). Her bowel and bladder function had gradually improved. Follow-up MRI and MRA, obtained 3 years after embolization, revealed no recurrent of the DAVF and diffuse mild spinal cord atrophy. The patient still had spastic paraparesis (MRC scale grade $0-1$ ). However, she could get back to the activities of daily living at home.

\section{Discussion}

Medullary congestion can be either due to intracranial DAVFs with caudally drainage into anterior spinal vein through the medullary veins, or spinal DAVFs with rostrally drainage into the medullary veins through anterior spinal vein. Congestion of the medulla may be the result of Cognard type $V$ intracranial dural arteriovenous fistulas, characterized by venous drainage into spinal perimedullary veins and the veins surrounding the brainstem [11]. Rarely, spinal vascular malformations at the level of lumbar or thoracic spine may cause remote brainstem symptoms from venous drainage extending to the intracranial veins. Wu, et al. [12] reported an elderly male with lumbar SDAVF at the level of L1 presenting with brainstem dysfunction. His clinical deterioration developed 3 days after admission. Despite successful endovascular treatment, there was no improvement in bulbar function and occurrence of aspiration pneumonia. He expired due to sudden apnea one month after treatment. Another case report by Cordato, et al. [13], a thoracic DAVF at the level of T4 caused remote mild brainstem symptoms in an elderly male. Surgical disconnection of the fistula from the normal venous outflow resulted in significant improvement of symptoms.

We reviewed the literature of lower cervical DAVFs, and found 9 cases, including our present case, with 10 fistulas due to bilateral lesions in one case (Table 1) $[10,14-20]$. The collected data in this review included demographic data (i.e. gender and age of patient), side of fistula, level of fistula, symptoms and signs of the patients, location of venous congestion on MRI, the presence of associating arterial or venous aneurysm on DSA or MRA, treatment of the fistulas, and neurological outcome after treatment. From the literature review, there were 6 males, and 3 females with median age of 42 years, ranging from 34 to 69 years. The fistulas were located at C4 nerve root in one case, C5 in 3, C6 in 2, C7 in 2, and C8 in 2. Six fistulas were located on the right side, and four on the left. Venous congestion involved brainstem, i.e. Medulla oblongata, pons, and/ or midbrain, in 3 cases, including our case. Most patients had progressive myelopathy and/or bowel and bladder dysfunction. Three patients with SAH were found to be associated with arterial or venous aneurysm. Two patients, including our case, developed respiratory dysfunction.

Lower cervical DAVF was firstly reported by Willinsky, et al. [14] since 1990. The diagnosis was delayed seven months after presentation of SAH in young male. He developed a progressive quadriparesis with bowel and bladder dysfunction, and MRI suggested a cervical intramedullary lesion. A laminectomy was performed and revealed an arteriovenous malformation on the cervical cord. DSA demonstrated DAVF on the right C8 nerve root, supplied from the right costocervical and vertebral arteries. The fistula drained medially into the intradural 


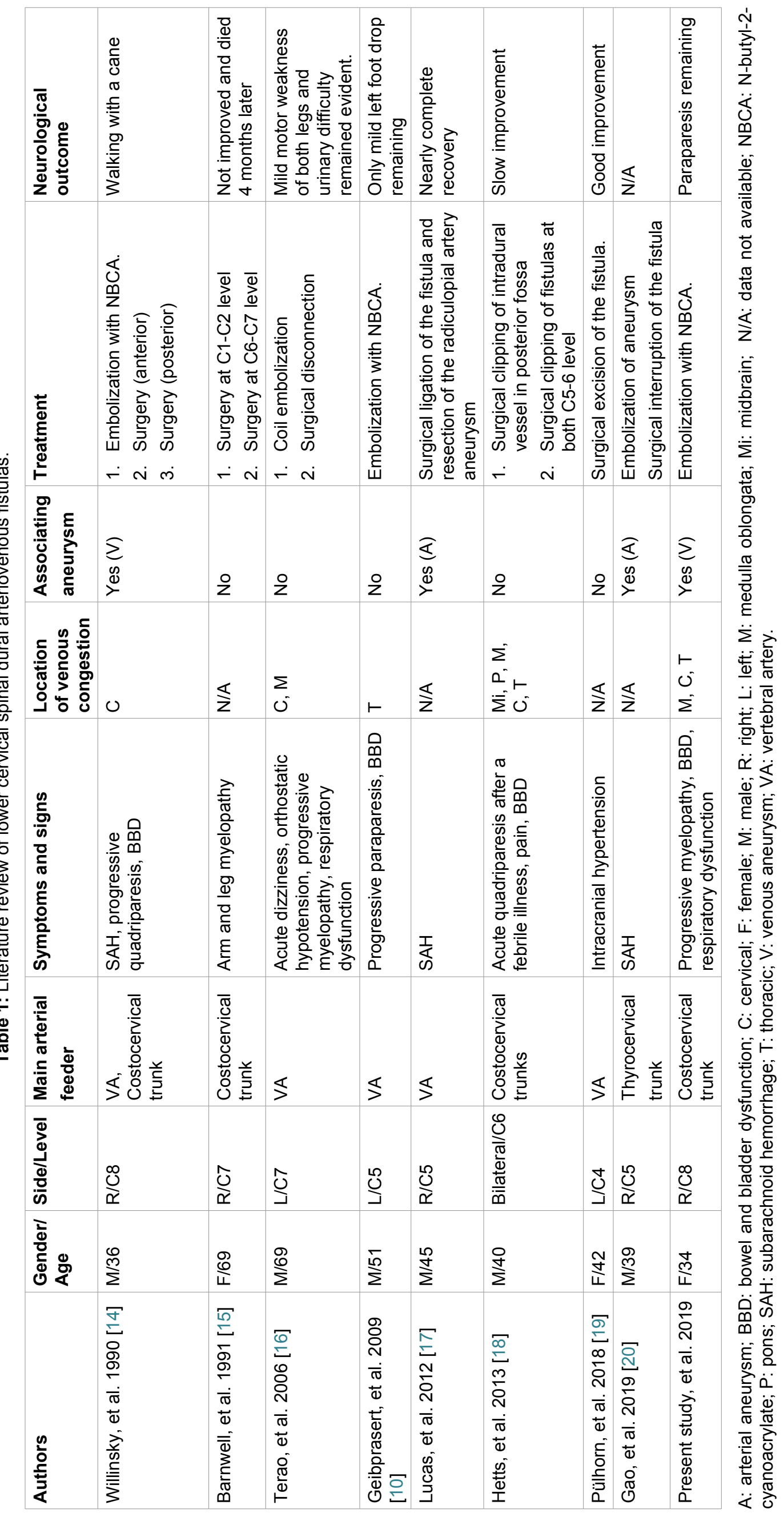


coronal veins with a large venous pouch. Two months after embolization with NBCA, the patient had clinical deterioration from remaining fistula. An anterior surgical approach failed to locate the fistula, and the right VA was ligated. The fistula still received from retrograde flow and was successfully treated by obliteration of the intradural vein from posterior approach. His symptoms had improved until walking with a cane. The following year, without demonstrated figure, Barnwell, et al. [15] reported a 69-year-old female presented arm and leg myelopathy. After coagulation of the first fistula at $\mathrm{C1}$ $\mathrm{C} 2$, follow-up angiography disclosed the second fistula at $\mathrm{C} 5-\mathrm{C} 6$, underwent surgical closing later. Her condition never improved and she died 4 months later. More than a decade later, Terao, et al. [16] reported a 69-year-old male presented with acute dizziness, orthostatic hypotension, progressive myelopathy, and urinary dysfunction. One month after initial symptoms, he developed rapid deterioration in consciousness and respiratory dysfunction, needed endotracheal intubation. MRI showed high signal intensity from the medulla oblongata to cervical spinal cord at the level of C6. The DAVF at the $\mathrm{C} 7$ nerve root was treated using coil embolization of the main feeder vessel from the left VA. Few months later, the patient developed the same previous clinical deterioration caused by recurrent DAVF. Proximal draining vein was interrupted surgically. One year after surgery, he remained mild motor weakness of both legs and urinary dysfunction. Few years later, Geibprasert, et al. [10] reported a cervical DAVF and clearly reviewed the literature by exclusion of other types of spinal vascular malformations, i.e., epidural arteriovenous shunts, radicular arteriovenous malformations, perimedullary AVFs. A middle-aged male presented with progressive paraparesis with bowel and bladder dysfunction. The fistula at the level of C5 was difficultly detected and successfully obliterated using NBCA. The patient had nearly complete recovery of the paraparesis with only a mild left foot drop remaining. Another few years later, Lucas, et al. [17] reported a middle-aged male presented with intracranial SAH. CT scan and MRI of the neck also showed an anterior subdural hematoma from C2-C4. He developed hydrocephalus and underwent a ventriculostomy. DSA demonstrated a cervical DAVF at the level of C5, supplied from the right VA. They suspected that a radiculopial artery aneurysm, probable mycotic type, at the same level from the right thyrocervical trunk was the source of SAH. The fistula and an aneurysm were successfully treated at the same setting by posterior surgical approach. Histological examination revealed no evidence of infection. They propose that the hemorrhage from lower cervical DAVF leaded to more aggressive clinical course than craniocervical DAVF. The following year, Hetts, et al. [18] reported bilateral lower cervical DAVFs arising at the same level associated with intracranial venous drainage in a 40-year-old male. The fistula was misidentified as a craniocervical DAVF, leading to surgical ligation of the bridging vein. Postoperative DSA disclosed redirection of blood flow to veins along the surface of the medulla, pons, and midbrain with drainage into the cavernous sinuses. The patient underwent a second curative surgery in the lower cervical spine, and slowly improved in extremity and bowel/ bladder dysfunction. Recently, Pülhorn, et al. [19] reported extremely rare presentation from cervical DAVF at the $\mathrm{C} 4$ exit root in middle-aged female. The patient fulfilled the criteria for diagnosis of idiopathic intracranial hypertension. After surgical obliteration of the fistula, her symptoms improved considerably on longterm follow-up. They speculated that impaired venous outflow from extracranial vascular malformations may probably cause intracranial hypertension. Regarding to recently teaching neuroimages by Gao, et al. [20], lower cervical DAVF presenting as SAH was demonstrated. The right-sided fistula was supplied by the right thyrocervical trunk and recruited additional spinal pial arteries from anterior spinal artery of the left VA. They speculated that a spinal pial aneurysm was potential cause of hemorrhage. Without clinical description, endovascular embolization of the aneurysm and surgical interruption of the fistula were performed successfully.

According to the anatomical study of Chakravorty, et al. [21] since 1971, the common radicular artery divides into anterior and posterior branches. Cervical radicular arteries usually run into the spinal canal through the intervertebral foramens accompanying the cervical nerve roots. These arteries may originate from VA, and subclavian branches, i.e., ascending cervical artery from the thyrocervical trunk, and deep cervical artery from the costocervical trunk. Cervical radicular arteries in the upper six segments can arise from the VA or the ascending cervical artery. For the seventh or eighth segments, it always arises from the deep cervical artery. Recently, Arslan, et al. [22] also studied in human cadaveric dissections and found that the cervical radicular arteries arise from the VA originating from its posterior aspect at each level, most often at the $\mathrm{C} 6$ level. The ascending cervical arteries give spinal branches to the C4 and C5 nerve roots. The deep cervical arteries always enter the C5-6, C6-7, and C7-T1 foramens and penetrated the dural sleeve within the posteroinferior portion of the foramen. In addition, the deep cervical artery may give off from the subclavian artery. Regarding our literature review, all fistulas, including our case, except one supplied from VA and/or costocervical trunk.

Understanding knowledge of the venous drainage of the spine and spinal cord is imperative and leads to correct diagnosis, localize the site of the fistula, and proper management [23]. Few studies demonstrated that misinterpretation resulted in misplace surgery, needed second operation, leading to delayed treatment and poor clinical outcomes $[15,18]$.

Spinal venous structures, forming complex networks, 
can be divided into intrinsic, extrinsic, and extradural systems. We previously explained about extradural venous system in another interesting case report [24]. The intrinsic venous system is composed of axial, longitudinal intrinsic, and transmedullary anastomosis. In the central region, capillaries from the symmetrical medial parts of both sides of gray matter drain axially into the ventral and dorsal sulcal veins. Additionally, capillaries from peripheral gray and white matter drain peripherally toward radial veins. The extrinsic venous system includes the pial venous networks (coronal venous plexus), longitudinally oriented extrinsic venous system (e.g., anterior and posterior spinal veins), and radicular veins. The anterior spinal vein, located subpially, accompanies the anterior spinal artery on the anteromedial surface of the spinal cord. The posterior spinal vein, located in subarachnoid space, may be single, double, or triple channel on the posteromedial or posterolateral surface. On the surface of the spinal cord, radial veins form a venous ring and subsequently anastomose with pial venous network and longitudinally oriented extrinsic venous system $[23,25,26]$. As clearly shown in our case, transmedullary anastomosis connected between the ventral and dorsal spinal cord veins. Radicular veins divided into ventral and dorsal veins. They may pierce the dura with the nerve root in $60 \%$, resulting in transdural portion of the vein that this feature represents an anti-reflux arrangement protecting the venous reflux of the spinal cord [25]. Radicular veins connect the extrinsic venous system to the extradural system [23].

The term of cerebrospinal venous system, anatomical and functional continuity between the intracranial veins and vertebral venous system, was proposed by Tobinick, et al. [27]. Due to valveless venous network results in bidirectional flow. Substantial anastomoses occur mainly in the suboccipital region as seen in the present study. The anterior medullary artery inferiorly communicates to the anterior spinal vein in the midline along anterior surface of the medulla and cervical spinal cord [28]. Interestingly, perimedullary anastomoses between engorged anterior spinal vein and lateral medullary vein at cervicomedullary junction on both sides were clearly demonstrated in our case. The veins surrounding brainstem are named based on the region (i.e., midbrain, pons, or medulla), location (e.g., median, lateral, etc.), and flow direction (i.e., transverse or longitudinal) of the brainstem. Transverse oriented veins include peduncular vein, vein of the pontomesencephalic sulcus, transverse pontine veins, vein of the pontomesencephalic sulcus, transverse medullary veins, etc. Longitudinal oriented veins include median anterior pontomesencephalic vein, median anterior medullary vein, lateral anterior pontomesencephalic vein, lateral anterior medullary vein, lateral mesencephalic vein, lateral medullary vein, etc. [29].

By cross the subarachnoid and subdural spaces, there are bridging veins directly draining the venous system around the brainstem to dural venous sinuses, including the posterior portion of the cavernous sinus, superior and inferior petrosal sinuses, sigmoid sinus, and marginal sinus. The petrosal veins represent bridging veins connecting between the veins of the brainstem and the petrosal sinuses, and divided into superior and inferior petrosal veins [29]. The medullary bridging veins mainly drain into inferior petrosal vein by joining between the vein of pontomesencephalic sulcus and lateral medullary veins. They can be connected with anterior condylar vein along the hypoglossal nerve, sigmoid sinus along the accessory nerve, a vein running along the first cervical root, or the marginal sinus [30]. Interestingly, Hetts, et al. [18] reviewed the venous drainage in the patient with bilateral lower cervical DAVFs arising at the same level. The right and left posterior radicular veins converge the enlarged posterior spinal vein, and rostrally run into the medial posterior medullary vein, exiting into the marginal sinus and epidural venous plexus through the bridging vein. The bridging vein was misinterpreted as a fistulous site, leading to misplace surgery. Another case report by Barnwell, et al. [15], they believed that there were two fistulas at C1-2 and C6-7 level. It is questionable that the fistula at C1-2 may be the venous drainage of the fistula at lower cervical level as mentioned earlier. In the present study, we can demonstrate the bridging vein connecting from the vein of pontomedullary sulcus to the inferior petrosal and sigmoid sinuses.

Clinical presentation is mainly influenced by venous drainage. Venous congestion is a major cause of neurological deterioration in SDAVFs by retrograde venous drainage into the valveless intrinsic veins of the spinal cord [31]. As mention earlier, lower cervical DAVFs can cause remote brainstem congestion, leading to aggressive manifestations including $\mathrm{SAH}$, rapidly progressive quadriparesis, and respiratory dysfunction $[14,16,17,20]$. SDAVFs commonly occur in the thoracolumbar region and clinical symptoms usually progress gradually [2]. In addition, most patients with lower cervical SDAVFs in our review had residual neurological deficits after treatment. Therefore, the natural history of DAVFs in lower cervical spine probably has more aggressive clinical course than thoracolumbar region.

SDAVFs can be treated by surgery, endovascular treatment, or both depend on institutions preference $[2,3]$. The goal of treatment is to obliterate the fistula, including the proximal portion of the draining radicular vein [1,32]. As found in the study of Terao, et al. [16], using coil embolization only in the main feeder vessel resulted in subsequent clinical deterioration due to recanalization of the fistula. From our review, most fistulas were successful treated by surgery using posterior approach [14,16-20]. However, cervical DAVF at the C8 nerve root as in our case can be successfully treated using transarterial embolization. For this location, it 
may be difficult to obliterate the fistula from anterior approach [14].

Interestingly, Enokizono, et al. [33] demonstrated the unique MRI findings of the black butterfly sign, characterized by symmetrical dark signals in the dorsal aspect of the medulla and the central gray matter of the spinal cord on susceptibility-weighted imaging (SWI) and GRE T2* WI. These findings probably represent hemorrhages caused by prolonged venous congestion. Similarly, MRI of the spine in our case also demonstrated the black butterfly sign on GRE T2*WI from cervical to upper thoracic cord, presumed to be the cause of permanent neurological deficit.

\section{Conclusion}

We reported an extremely rare case of progressive venous congestion of the medulla oblongata caused by the lower cervical DAVF, resulting in life-threatening condition. We speculated that SDAVFs at lower cervical region have more aggressive behavior than thoracolumbar region. Prompt treatment should be performed before clinical deterioration, especially brainstem dysfunction. Image studies should be careful interpreted to localize the exact location of the fistula to avoid misplace treatment, leading to delay in proper treatment resulting in poor neurological outcome.

\section{References}

1. Anson JA, Spetzler RF (1992) Interventional neuroradiology for spinal pathology. Clin Neurosurg 39: 388-417.

2. Spetzler RF, Detwiler PW, Riina HA, Porter RW (2002) Modified classification of spinal cord vascular lesions. J Neurosurg 96: 145-156.

3. Krings T, Lasjaunias PL, Geibprasert S, Hans FJ, Thron AK, et al. (2009) Classification of Spinal Vascular Malformations. The Neuroradiology Journal 22: 97-106.

4. lampreechakul $P$, Lertbutsayanukul $P$, Siriwimonmas $S$, Jittapiromsak P, Tantivatana J, et al. (2019) Persistent central neuropathic pain caused by intramedullary hemorrhage from spinal dural arteriovenous fistula: A case report and literature review. Austin J Anesthesia and Analgesia 7: 1-7.

5. Kinouchi H, Mizoi K, Takahashi A, Nagamine Y, Koshu K, et al. (1998) Dural arteriovenous shunts at the craniocervical junction. J Neurosurg 89: 755-761.

6. Aviv RI, Shad A, Tomlinson G, Niemann D, Teddy PJ, et al. (2004) Cervical dural arteriovenous fistulae manifesting as subarachnoid hemorrhage: Report of two cases and literature review. AJNR Am J Neuroradiol 25: 854-858.

7. Kai Y, Hamada J, Morioka M, Yano S, Mizuno T, et al. (2005) Arteriovenous fistulas at the cervicomedullary junction presenting with subarachnoid hemorrhage: Six case reports with special reference to the angiographic pattern of venous drainage. AJNR Am J Neuroradiol 26: 1949-1954.

8. Kulwin C, Bohnstedt BN, Scott JA, Cohen-Gadol A (2012) Dural arteriovenous fistulas presenting with brainstem dysfunction: diagnosis and surgical treatment. Neurosurg Focus 32: E10.
9. Wu Q, Wang HD, Shin YS, Zhang X (2014) Brainstem Congestion due to Dural Ateriovenous Fistula at the Craniocervical Junction. J Korean Neurosurg Soc 55: 152155.

10. Geibprasert S, Pongpech S, Jiarakongmun P, Krings T (2009) Cervical spine dural arteriovenous fistula presenting with congestive myelopathy of the conus. J Neurosurg Spine 11: 427-431.

11. Copelan AZ, Krishnan A, Marin H, Silbergleit R (2018) Dural Arteriovenous Fistulas: A Characteristic Pattern of Edema and Enhancement of the Medulla on MRI. AJNR Am J Neuroradiol 39: 238-244.

12. Wu SL, Tsui HW, Chuang YC, Kuo HC, Chen CJ (2006) Lumbar spinal dural arteriovenous fistula presenting with brainstem symptomatology: A case report. Acta Radiol 47: 730-732.

13. Cordato DJ, Davies MA, Masters LT, Cremer PD, Schwartz RS, et al. (2004) Positional vomiting due to a thoracic spinal dural arteriovenous fistula. Case report. J Neurosurg Spine 1: $219-222$.

14. Willinsky R, TerBrugge $K$, Lasjaunias $P$, Montanera W (1990) The variable presentations of craniocervical and cervical dural arteriovenous malformations. Surg Neurol 34: 118-123

15. Barnwell SL, Halbach VV, Dowd CF, Higashida RT, Hieshima GB, et al. (1991) Multiple dural arteriovenous fistulas of the cranium and spine. AJNR Am J Neuroradiol 12: $441-445$.

16. Terao $T$, Taniguchi $M$, Ide $K$, Shinozaki $M$, Takahashi $H$ (2006) Cervical dural arteriovenous fistula presenting with brainstem dysfunction: case report and review. Spine 31: E722-E727.

17. Lucas JW, Jones J, Farin A, Kim P, Giannotta SL (2012) Cervical spine dural arteriovenous fistula with coexisting spinal radiculopial artery aneurysm presenting as subarachnoid hemorrhage: Case report. Neurosurgery 70 : E259-E263.

18. Hetts SW, English JD, Stiver SI, Singh V, Yee EJ, et al. (2013) Bilateral cervical spinal dural arteriovenous fistulas with intracranial venous drainage mimicking a foramen magnum dural arteriovenous fistula. Interv Neuroradiol 19: 483-488.

19. Pülhorn $H$, Chandran $A$, Nahser $H$, Wilby MJ, McMahon C (2018) Intracranial Hypertension Secondary to Cervical Dural Arteriovenous Fistula. Asian J Neurosurg 13: 854857.

20. Gao P, Du S, Ren J, Li G, Zhang H (2019) Teaching Neurolmages: Lower cervical spine dural arteriovenous fistula presenting as subarachnoid hemorrhage. Neurology 92: e1798-e1800.

21. Chakravorty BG (1971) Arterial supply of the cervical spinal cord (with special reference to the radicular arteries). Anat Rec 170: 311-329.

22. Arslan M, Acar HI, Comert A, Tubbs RS (2018) The cervical arteries: an anatomical study with application to avoid the nerve root and spinal cord blood supply. Turk Neurosurg 28: $234-240$

23. Griessenauer CJ, Raborn J, Foreman P, Shoja MM, Loukas $M$, et al. (2015) Venous drainage of the spine and spinal cord: a comprehensive review of its history, embryology, anatomy, physiology, and pathology. Clin Anat 28: 75-87.

24. lampreechakul $P$, Liengudom $A$, Wangtanaphat $K$, Lertbut- 
sayanukul $P$, Wattanasen $Y$, et al. (2018) progressive cervical myelopathy caused by bilateral spontaneous high-flow vertebro-vertebral arteriovenous fistulas associated with fibromuscular dysplasia successfully treated by endovascular v2 segments sacrifice using fibered coils: A case report. Clin Med Rev Case Rep 5: 1-12.

25. Lasjaunias P, Berenstein A, Ter Brugge KG (2001) Spinal and Spinal Cord Arteries and Veins. In: Lasjaunias $P$, Berenstein A, Ter Brugge KG, Surgical Neuroangiography. Volume 1, Springer, Berlin, Heidelberg, 73-164.

26. Miyasaka K, Asano T, Ushikoshi S, Hida K, Koyanagi I (2000) Vascular anatomy of the spinal cord and classification of spinal arteriovenous malformations. Interv Neuroradiol 6: 195-198.

27. Tobinick E, Vega CP (2006) The cerebrospinal venous system: anatomy, physiology, and clinical implications. MedGenMed 8: 53.

28. Kiyosue H, Tanoue S, Sagara Y, Hori Y, Okahara M, et al. (2008) The anterior medullary-anterior pontomesencephalic venous system and its bridging veins communicating to the dural sinuses: Normal anatomy and drainage routes from dural arteriovenous fistulas. Neuroradiology 50: 1013-1023.

29. Rhoton AL (2000) The posterior fossa veins. Neurosurgery 47: S69-S92.

30. Baltsavias G, Parthasarathi V, Aydin E, Al Schameri RA, Roth P, et al. (2015) Cranial dural arteriovenous shunts. Part 1. Anatomy and embryology of the bridging and emissary veins. Neurosurg Rev 38: 253-263.

31. Kataoka $H$, Miyamoto $S$, Nagata I, Ueba T, Hashimoto $N$ (2001) Venous congestion is a major cause of neurological deterioration in spinal arteriovenous malformations. Neurosurgery 48: 1224-1229.

32. Jellema K, Sluzewski M, van Rooij WJ, Tijssen CC, Beute GN (2005) Embolization of spinal dural arteriovenous fistulas: importance of occlusion of the draining vein. $J$ Neurosurg Spine 2: 580-583.

33. Enokizono $M$, Sato $N$, Morikawa $M$, Kimura $Y$, Sugiyama A, et al. (2017) "Black butterfly" sign on T2*-weighted and susceptibility-weighted imaging: A novel finding of chronic venous congestion of the brain stem and spinal cord associated with dural arteriovenous fistulas. J Neurol Sci 379: 64-68. 